COLLOQUIUM MATHEMATICUM VOL. VIII 1961

\title{
SUR DEUX GENRES D'ESPACES COMPLETS
}

PAR

A. LELEK (WROCEAW)

Knaster [1] a attiré l'attention sur l'existence de deux genres topologiquement distincts d'espaces complets (dits aussi $\boldsymbol{G}_{\delta}$ absolus ou $\boldsymbol{G}_{\delta}$ dans un espace compact), e'est-à-dire qui sont homéomorphes à des espaces métriques complets.

$\Pi 1$ a appelé un espace complet $X$ de $I$ genre ([1], p. 264) lorsqu'il existe une homéomorphie de $X$ en sous-ensemble d'un espace $Y$ compact, $h: X \rightarrow Y$, telle que

$$
\begin{aligned}
& 1^{\circ} h(X)=\bigcap_{i=1}^{\infty} G_{i}, \\
& 2^{\circ} \operatorname{dim} \operatorname{Fr}\left(G_{i}\right)<\operatorname{dim} X,
\end{aligned}
$$

où $G_{i}$ est un ensemble ouvert dans $Y$ pour tout $i=1,2, \ldots$ Un espace complet $X$ est de II genre lorsqu'il ne se laisse pas représenter de cette façon.

9 désignant le segment $0 \leqslant x \leqslant 1$ de nombres réels et $\Upsilon$ l'ensemble des nombres irrationnels de $\mathcal{T}$, Knaster a montré, entre autres, que le produit cartésien $\chi \times 9$ est un espace complet de II genre ([1], p. 263-264) et posé la question s'il en est de même des espaces $\chi \times \Im^{n}$ pour tout $n>\mathbf{1}$ ([1], p. $\left.267,3^{\circ}\right)$. La réponse est affirmative en vertu du théorème 3 qui va suivre (voir p. 34, corollaire).

Le but de cette communication est de le démontrer et d'envisager quelques problèmes qui s'y rattachent.

1. Compactification. Appelons compactification d'un espace topologique $X$ toute homéomorphie $h_{c}: X \rightarrow \bar{X}$, où $\bar{X}$ est un espace compact tel que $h_{c}(X)$ est dense dans lui.

THÉ́RÈmE 1. Pour qu'un espace complet $X$ de dimension finie soit de I genre, il faut et il suffit qu'il existe une compactification $h_{c}: X \rightarrow \bar{X}$ telle que $\operatorname{dim}\left[\bar{X}-h_{c}(X)\right]<\operatorname{dim} X$. 
Démonstration. En admettant l'existence d'une telle compactification $h_{c}$ et $X$ étant complet, l'image $h_{c}(X)$ est complet, donc un $\boldsymbol{G}_{\delta}$ dans $\bar{X}\left([2]\right.$, p. 337), done de la forme $h_{c}(X)=\bigcap_{i=1}^{\infty} G_{i}$, où $G_{i}$ est ouvert dans $\bar{X}$ pour $i=1,2, \ldots$ On a par conséquent $\operatorname{Fr}\left(G_{i}\right)=G_{i}-G_{i} \subset \bar{X}-h_{c}(X)$, d'où $\operatorname{dim} \operatorname{Fr}\left(G_{i}\right) \leqslant \operatorname{dim}\left[\bar{X}-h_{c}(X)\right]<\operatorname{dim} X$ en vertu de l'hypothèse sur $h_{c}$. On a ainsi $1^{\circ}$ et $2^{\circ}$; la condition est done suffisante pour que $X$ soit de I genre.

Réciproquement, en admettant que $X$ est de I genre, les conditions $1^{\circ}$ et $2^{\circ}$ sont satisfaites pour un espace compact $Y$, une homéomorphie $h: X \rightarrow Y$ et une suite $G_{1}, G_{2}, \ldots$ d'ensembles ouvertes dans $Y$. Posons $h_{c}=h$ et $\bar{X}=\overline{h(X)}$ (fermeture dans $\bar{Y}$ ). On a

$$
\begin{aligned}
\vec{X}-h_{c}(X) & =\overline{h(X)}-h(X)=\overline{h(X)}-\bigcap_{i=1}^{\infty} G_{i}=\bigcup_{i=1}^{\infty}\left[\overline{h(X)}-G_{i}\right] \\
& \subset \bigcup_{i=1}^{\infty}\left(G_{i}-G_{i}\right)=\bigcup_{i=1}^{\infty} \operatorname{Fr}\left(G_{i}\right),
\end{aligned}
$$

car $\overline{h(\bar{X})} \subset \overline{G_{i}}$ pour $i=1,2, \ldots$ en vertu de $1^{\circ}$. Il en résulte, en posant

$$
A_{i}=\operatorname{Fr}\left(G_{i}\right) \frown\left[\bar{X}-h_{c}(X)\right] \text {, }
$$

que

$$
\bar{X}-h_{c}(X)=\bigcup_{i=1}^{\infty} A_{i}
$$

En vertu de (1), les ensembles $A_{i}$ sont fermés dans $\bar{X}-h_{c}(X)$ et on a $A_{i} \subset \operatorname{Fr}\left(G_{i}\right)$, d'où $\operatorname{dim} A_{i} \leqslant \operatorname{dim} \operatorname{Fr}\left(G_{i}\right)<\operatorname{dim} X$ en vertu de $2^{\circ} . \operatorname{Vu}(2)$, on a par conséquent (voir [2], p. 176) $\operatorname{dim}\left[\bar{X}-h_{c}(X)\right] \leqslant \operatorname{dim} A_{i}<\operatorname{dim} X$; la condition est done nécessaire.

2. Espaces complets de I genre. Il s'ensuit aussitôt du théorème 1 que tout espace complet de I genre et de dimension. 0 est compact (cf. [1], p. 263). Pour tout $n>0$, il existe cependant des $X$ complets do dimension $n$ qui sont de I genre et qui ne sont même pas des $\boldsymbol{F}_{\sigma}$ absolus (c'est-à-dire des $\boldsymbol{F}_{\sigma}$ dans un espace compact, ou encore - ce qui revient au même des sommes dénombrables d'ensembles compacts). Lin posant par exemple $X=9^{n+1}-(9-\Upsilon)^{n+1}$, on a notoirement $\operatorname{dim} X=n, \bar{X}=9^{n+1}$ et, le complément $(9-\Upsilon)^{n+1}$ de $X$ à $9^{n+1}$ étant dénombrable, il vient $\operatorname{dim}(9-\Upsilon)^{n+1}=0<\operatorname{dim} X$. Ainsi, vu le théorème $1, X$ est complet de I genre et n'est pas un $F_{\sigma}$ dans $\mathscr{S}^{n+1}$ (en vertu du théorème de Baire).

Il existe aussi des $X$ complets de dimension infinie qui sont de I genre sans être des $\boldsymbol{F}_{\sigma}$ absolus. Soit par exemple $X=9^{\mathrm{N}_{0}}-g(9-\Upsilon)$ où $g: \mathcal{G} \rightarrow \mathcal{G}^{\mathrm{N}_{0}}$ est une homéomorphie du segment $g$ en un sous-ensemble du eube $9^{N_{0}}$ de Hilbert. Le complément $g(\Im-\Upsilon)$ de $X$ à $\mathcal{9}^{*}$ étant dé- nombrable, on a $g(\mathscr{S}-\Upsilon)=\left\{p_{1}, p_{2}, \ldots\right\}$, d'où $X=\bigcap_{i=1}^{\infty} G_{i}$ où $G_{i}=\mathscr{S}^{\boldsymbol{N}_{0}}-$ $-\left(p_{i}\right)$ pour $i=1,2, \ldots$ Il en résulte que $\operatorname{dim} \operatorname{Fr}\left(G_{i}\right)=\operatorname{dim}\left(p_{i}\right)=0$ $<\operatorname{dim} X$ pour $i=1,2, \ldots$; done les conditions $1^{\circ}$ et $2^{\circ}$ sont satisfaites en posant $Y=\mathscr{G}^{N_{0}}$ et $h=$ identité, et $X$ est complet de I genre. $X$ n'est pas un $F_{\sigma}$ dans $g^{\aleph_{0}}$, car dans le cas contraire l'ensemble $X \cap g(\Im)$ $=g(\Im)-g(\Im-\Upsilon)=g(\Upsilon)$ serait alors un $\boldsymbol{F}_{\sigma}$ absolu, ce qui est impossible, $g$ étant une homéomorphie.

3. Espaces complets lacunaires. Je dis qu'un espace topologique $X$ est lacunaire lorsque tout ensemble compact $F \subset X$ y est un ensemble frontière (c'est-à-dire que $\overline{X-F}=X$ ). L'ensemble $\chi$ par exemple est un espace lacunaire.

THÉOR亡̀ME 2. $X$ étant un espace métrique compact, soient $A$ un sousensemble de $X, Y$ un espace complet lacunaire et $f: A \rightarrow Y$ une fonction continue telle que $f(A)=Y, 0 \leqslant n \leqslant \operatorname{dim} f^{-1}(y)$ et que $f^{-1}(y)$ soit compact pour tout $y \in \bar{Y}$. Alors on a de même $n \leqslant \operatorname{dim}(X-A)$.

Démonstration. Supposons que l'on ait $\operatorname{dim}(X-A) \leqslant n-1$, donc (voir [2], p. 182) que $X-A$ satisfasse à la condition $D_{n-1}$. Il en résulte, l'espace métrique $X$ étant compact par hypothèse, qu'il y existe pour tout $j=1,2, \ldots$ une somme finie $H_{j}=H_{j 0} \cup H_{j 1} \cup \ldots \cup H_{j m_{j}}$ d'ensembles ouverts, tels que

$$
\begin{gathered}
X-A \subset H_{j}, \\
\delta\left(H_{j i}\right)<j^{-1} \quad \operatorname{pour} \quad i=0,1, \ldots, m_{j}, \\
i_{0}<i_{1}<\ldots<i_{n} \leqslant m_{j} \text { entraîne } H_{j i_{0}} \cap H_{j i_{1}} \cap \ldots \cap H_{j i_{n}}=0
\end{gathered}
$$

(voir [2], p. 184). $X-H_{j}$ est donc compact et on a $X-H_{j} \subset A$ en vertu de (3). Par conséquent, $f\left(X-H_{j}\right)$ est compact et non-dense dans $Y$, $f$ étant continue et $Y$ lacunaire. Ainsi, l'ensemble $S=\bigcup_{j=1}^{\infty} f\left(X-H_{j}\right)$ est de I catégorie dans $Y$, d'où l'existence d'un point $y_{0} \in Y-S$ en vertu du théorème de Baire. On en conclut que

$$
\begin{gathered}
f^{-1}\left(y_{0}\right) \subset f^{-1}(Y-S)=f^{-1}(Y)-f^{-1}(S)=A-\bigcup_{j=1}^{\infty} f^{-1} f\left(X-H_{j}\right) \\
\subset X-\bigcup_{j=1}^{\infty}\left(X-H_{j}\right)=\bigcap_{j=1}^{\infty} H_{j} .
\end{gathered}
$$

Il s'ensuit en vertu de (4) et (5) grâce à la compacité de $f^{-1}\left(y_{0}\right)$ que cet ensemble satisfait à la condition $D_{n-1}$, d'où (voir $[3]$, p. 72) $\operatorname{dim} f^{-1}\left(y_{0}\right)$ $\leqslant n-1$ contrairement à l'hypothèse.

Colloquium Mathematicum viII 
4. Espaces complets de III genre. On a le théorème suivant:

THÉoRÈnE 3. Quel que soit l'espace compact $Z$ de dimension $n \geqslant 0$,

Démonstration. On a $\operatorname{dim} \Upsilon \times Z=\operatorname{dim} Z=n, \operatorname{car} \operatorname{dim} \Upsilon=0$. Soit $h_{c}: \Upsilon \times Z \rightarrow \overline{\Upsilon \times Z}$ une compactification de $\Re \times Z$. Considérons la projection $p(\xi, z)=\xi$ pour tout $\xi \dot{\epsilon} \Upsilon$ et $z \epsilon Z$, d'où $p: \Upsilon \times Z \rightarrow \Upsilon$ et $p(\Upsilon \times Z)=\chi$, et la fonction continue $f=p h_{c}^{-1}$, d'où $f: h_{c}(\Upsilon \times Z) \rightarrow \Upsilon$ et $f h_{c}(\eta \times Z)=\chi$

Il en résulte que pour tout $\xi \epsilon \bigcap$ on a

$$
f^{-1}(\xi)=\left(p h_{c}^{-1}\right)^{-1}(\xi)=h_{c} p^{-1}(\xi)=h_{c}[(\xi) \times Z],
$$

d'où $\operatorname{dim} f^{-1}(\xi)=\operatorname{dim} Z=n$ et $f^{-1}(\xi)$ est compact. En posant $X=\overline{\Upsilon \times Z}, \quad A=h_{c}(\Re \times Z)$ et $Y=\chi$, on a done $n \leqslant \operatorname{dim}[\Upsilon \times \bar{Z}-$ $\left.-h_{c}(\Re \times Z)\right]$ en vertu du théorème 2 et, par conséquent, l'espace complet $\Upsilon \times Z$ n'est pas de $\Upsilon$ genre en vertu du théorème 1 .

Corollame. Tout $\boldsymbol{G}_{\delta}$ de la forme $\chi \times \mathscr{S}^{n}$ où $n>0$ est de II genre.

L'existence des espaces métrisables séparables et complets de II genre et de toute dimension finie étant ainsi établie, les questions suivantes s'imposent:

P 312. Existe-t-il un espace métrisable séparable et complet de II genre qui soit de dimension infinie?

P 313. Existe-t-il, pour tout espace $X$ métrisable séparable complet de II genre et de dimension positive finie, un espace compact $Z$ de dimension positive, tel que le produit cartésien $\chi \times Z$ ait une image homéomorphe dans $X$ ?

\section{TRA VAUX OITÉS}

[1] B. Knaster, Un théorème sur la compactification, Annales de la Société Polonaise de Mathématique 25 (1952), p. 252 - 267.

[2] C. Kuratowski, Topologie I, Warszawa 1952.

[3] - Topologie II, Warszawa 1952.

INSTITUT MATHÉMATIQUE DE L'UNIVERSITE DE WROCLAW

Reģu par la Rédaction le 1. 12.1959

UNE GÉNÉRALISATION DU THÉORËME DE KURATOWSKI

SUR LA CARACTÉRISATION MÉTRIQUE DE LA RÉTRACTION

\section{PAR}

W. NITKA (WROCEAW)

Soient $M$ un espace métrique, $\varrho$ la distance dans $M$ et $R \subset M$. Pour tout point $x \in M$, soit $\varrho(x, R)=\inf _{y_{\epsilon} R} \varrho(x, y)$ la distance entre $x$ et $R$.

Appelons convexité de $R$ relative à $\varrho$ au sens de de Groot [2] la propriété métrique suivante de $R$ :

(G) $x \in R, y \in R, z \in M$ et $\varrho(x, z)+\varrho(z, y)=\varrho(x, y)$ entraînent $z \epsilon R$.

$R$ est dit un rétracte de $M$ (notion due à Borsuk [1]) lorsqu'il existe une fonction continue $r: M \rightarrow R$ (dite rétraction) telle que $r(y)=y$ pour tout $y \in R$.

Considérons encore la propriété métrique suivante d'un $R \subset M$ relative à $\varrho$ :

(K) il existe une fonction $r: M \rightarrow R$ faisant correspondre à tout point $x \in M$ un point $r(x) \in R$ tel que $\varrho(x, R)=\varrho(x, r(x))$.

Kuratowski [4] a démontré que

(1) si $M$ est compact, $R$ fermé et $R$ possède la propriété (K) relativement à $\varrho$, la fonction $r$ dont il y est question est continue ( $R$ est donc un rétracte de $M$ );

(2) réciproquement, si $R$ est un rétracte de $M$, il existe dans $M$ une métrique $\varrho^{*}$ topologiquement équivalente à $\varrho$ (c'est-à-dire que toute suite de points de $M$ qui est convergente dans $\varrho$ l'est dans $\varrho^{*}$ et réciproquement) et telle que $R$ possède la propriété (K) relativement à $\varrho^{*}$.

Il suffit d'ailleurs dans (1) de ne supposer que la compacité de $R$.

Convenons de dire qu'une métrique est conforme à la topologie d'un espace dans lequel elle est définie lorsque la convergence d'une suite quelconque de ses points, d'après sa topologie, vers l'un de ses points équivaut à celle vers le même point d'après la métrique en question.

On a la généralisation suivante de (2): 